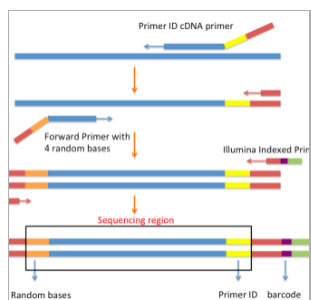

VERSION 3

AUG 12, 2018

\section{OPEN ठैACCESS}

DOI:

dx.doi.org/10.17504/protocol s. io.sjdeci6 Protocol Citation: Shuntai Zhou 2018. Primer ID MiSeq Library Prep. protocols.io rotocols.io.sjdeci6

License: This is an open access protocol distributed under the terms of the Creative Commons the Creative Commons Attribution License, which permits unrestricted use, distribution, and reproduction in any medium, provided the original author and source are credited

Protocol status: Working We use this protocol and it's working

Created: Aug 11, 2018

Last Modified: Aug 12, 2018 PROTOCOL integer ID: 14661

Keywords: NGS, Primer ID
(3) Primer ID MiSeq Library Prep V.3

Shuntai Zhou ${ }^{1}$

${ }^{1}$ University of North Carolina at Chapel Hill

Shuntai Zhou

\section{ABSTRACT}

This is the protocol to prepare Primer ID MiSeq sequencing library. Viral RNA was first extracted using QIAamp viral RNA extraction kit. The block of random nucleotides (Ns) in the cDNA primers served as the Primer ID. The Superscript III kit was used for the cDNA synthesis. We used two rounds of PCR to amplify the CDNA and incorporate Illumina indexed adapters with KAPA2G Robust and KAPA HiFi PCR kits, respectively.

\section{ATTACHMENTS \\ amplicon \\ protocol_v1.5.docx \\ GUIDELINES}

Viral RNA extraction using QIAamp vRNA mini kit.

\section{Primer ID cDNA primer}

cDNA synthesis

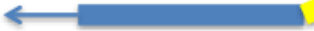

$$
\downarrow
$$

$1^{\text {st }}$ Round PCR

Forward Primer with 4 random bases

Illumina Indexed Primer

$2^{\text {nd }}$ Round PCR
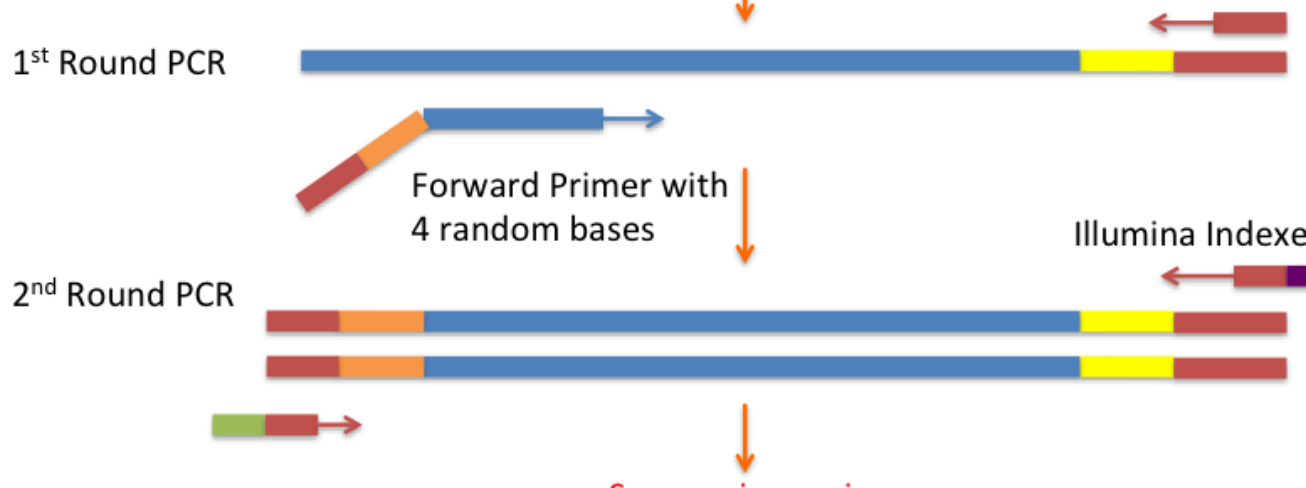

Sequencing region

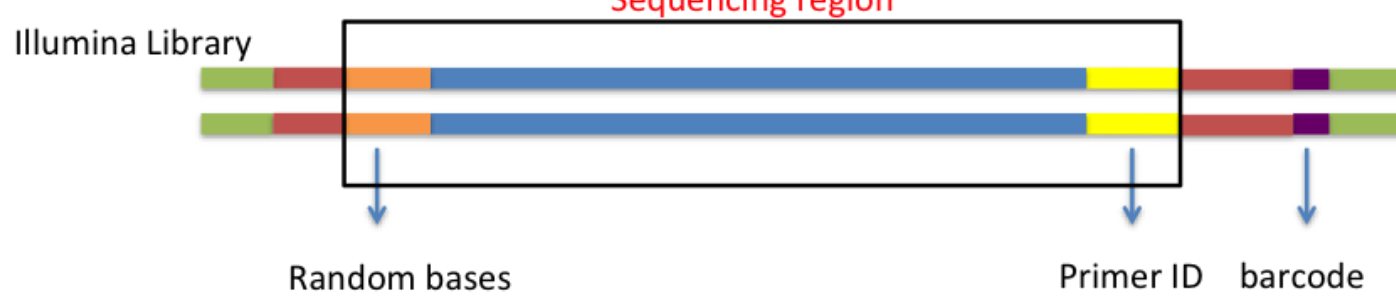

Primers (NOTE: HIV-1 ENV REGION AS AN EXAMPLE, SUBSTITUTE THE BLUE Sequences in the forward primer and cDNA primer) 


\begin{tabular}{|l|l|}
\hline V1F(forward) 5'-3' & $\begin{array}{l}\text { GCCTCCCTCGCGCCATCAGAGATGTGTATAAGAGACAGNNNNTTATGGGATC } \\
\text { AAACCTAAAGCCATGTGTA }\end{array}$ \\
\hline $\begin{array}{l}\text { BV3R Uni (cDNA } \\
\text { Primer) 5'-3' }\end{array}$ & GTGACTGGAGTTCAGACGTGTGCTCTTCCGATCT NN NNN NN NNN N CAGTCCATTT \\
\hline & TGCTCTACTAATGTTACAATGTGC \\
\hline Universal Adapter & GATGATACGGCGACCACCGAGATCTACACGCCTCCCTCGCGCCATCAGAGATGT \\
\hline Indexed Adapter & \begin{tabular}{l} 
CAAGCAGAAGACGGCATACGAGATNNNNNNGTGACTGGAGTCAGACGTGT \\
\hline ADPT_2a
\end{tabular} \\
\hline
\end{tabular}

Note: Primer ID primer and forward primer use random bases. Indexed primers have 24 fixed barcodes.

Table of Indexed Primers

\begin{tabular}{|c|c|c|c|}
\hline Indexed Primer & Index & $\begin{array}{c}\text { Index } \\
\text { Sequence }\end{array}$ & Sequence \\
\hline PCR Primer, Index 1 & 1 & ATCACGA & CAAGCAGAAGACGGCATACGAGATCGTGATGTGACTGGAGTTCAGACGTGTGCTC \\
\hline PCR Primer, Index 2 & 2 & CGATGTA & CAAGCAGAAGACGGCATACGAGATACATCGGTGACTGGAGTTCAGACGTGTGCTC \\
\hline PCR Primer, Index 3 & 3 & TTAGGCA & CAAGCAGAAGACGGCATACGAGATGCCTAAGTGACTGGAGTTCAGACGTGTGCTC \\
\hline PCR Primer, Index 4 & 4 & TGACCAA & CAAGCAGAAGACGGCATACGAGATTGGTCAGTGACTGGAGTTCAGACGTGTGCTC \\
\hline PCR Primer, Index 5 & 5 & ACAGTGA & CAAGCAGAAGACGGCATACGAGATCACTGTGTGACTGGAGTTCAGACGTGTGCTC \\
\hline PCR Primer, Index 6 & 6 & GCCAATA & CAAGCAGAAGACGGCATACGAGATATTGGCGTGACTGGAGTTCAGACGTGTGCTC \\
\hline PCR Primer, Index & 7 & CAGATCA & CAAGCAGAAGACGGCATACGAGATGATCTGGTGACTGGAGTTCAGACGTGTGCTC \\
\hline PCR Primer, Index 8 & 8 & ACTTGAA & CAAGCAGAAGACGGCATACGAGATTCAAGTGTGACTGGAGTTCAGACGTGTGCTC \\
\hline PCR Primer, Index 9 & 9 & GATCAGA & CAAGCAGAAGACGGCATACGAGATCTGATCGTGACTGGAGTTCAGACGTGTGCTC \\
\hline PCR Primer, Index 10 & 10 & TAGCTTA & CAAGCAGAAGACGGCATACGAGATAAGCTAGTGACTGGAGTTCAGACGTGTGCTC \\
\hline PCR Primer, Index 11 & 11 & GGCTACA & CAAGCAGAAGACGGCATACGAGATGTAGCCGTGACTGGAGTTCAGACGTGTGCTC \\
\hline PCR Primer, Index 12 & 12 & CTTGTAA & CAAGCAGAAGACGGCATACGAGATTACAAGGTGACTGGAGTTCAGACGTGTGCTC \\
\hline PCR Primer, Index 13 & 13 & TCCATAA & CAAGCAGAAGACGGCATACGAGATTATGGAGTGACTGGAGTTCAGACGTGTGCTC \\
\hline PCR Primer, Index 14 & 14 & GTACTAA & CAAGCAGAAGACGGCATACGAGATTAGTACGTGACTGGAGTTCAGACGTGTGCTC \\
\hline PCR Primer, Index 15 & 15 & ACAGTAA & CAAGCAGAAGACGGCATACGAGATTACTGTGTGACTGGAGTTCAGACGTGTGCTC \\
\hline PCR Primer, Index 16 & 16 & CTCATGA & CAAGCAGAAGACGGCATACGAGATCATGAGGTGACTGGAGTTCAGACGTGTGCTC \\
\hline PCR Primer, Index 17 & 17 & ACGATAA & CAAGCAGAAGACGGCATACGAGATTATCGTGTGACTGGAGTTCAGACGTGTGCTC \\
\hline PCR Primer, Index 18 & 18 & TGCAGAA & CAAGCAGAAGACGGCATACGAGATTCTGCAGTGACTGGAGTTCAGACGTGTGCTC \\
\hline PCR Primer, Index 19 & 19 & TTCATAA & CAAGCAGAAGACGGCATACGAGATTATGAAGTGACTGGAGTTCAGACGTGTGCTC \\
\hline PCR Primer, Index 20 & 20 & TGCTGTA & CAAGCAGAAGACGGCATACGAGATACAGCAGTGACTGGAGTTCAGACGTGTGCTC \\
\hline PCR Primer, Index 21 & 21 & TATCACA & CAAGCAGAAGACGGCATACGAGATGTGATAGTGACTGGAGTTCAGACGTGTGCTC \\
\hline PCR Primer, Index 22 & 22 & TGGATAA & CAAGCAGAAGACGGCATACGAGATTATCCAGTGACTGGAGTTCAGACGTGTGCTC \\
\hline PCR Primer, Index 23 & 23 & CGCATTA & CAAGCAGAAGACGGCATACGAGATAATGCGGTGACTGGAGTTCAGACGTGTGCTC \\
\hline PCR Primer, Index 24 & 24 & GCCTTAA & CAAGCAGAAGACGGCATACGAGATTAAGGCGTGACTGGAGTTCAGACGTGTGCTC \\
\hline
\end{tabular}

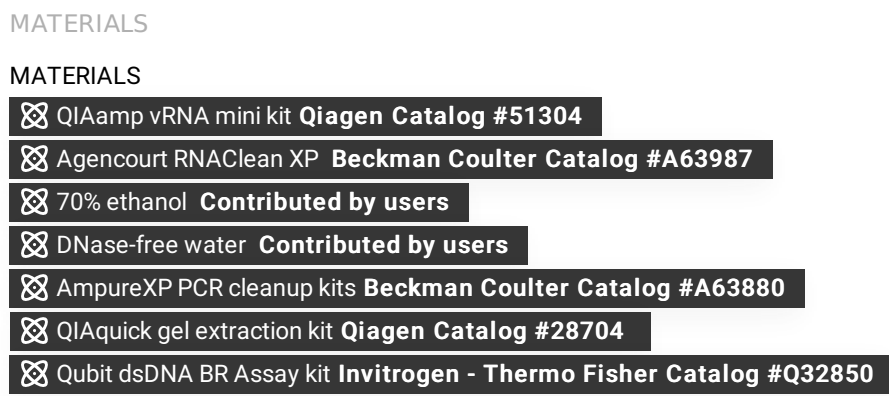

Prepare Primer Mix (Optional, only for multiplexed Primer ID library prep)

1 For multiplexing sequencing, first, prepare Primer Mix. Example (For HIV drug resistance pipeline). 


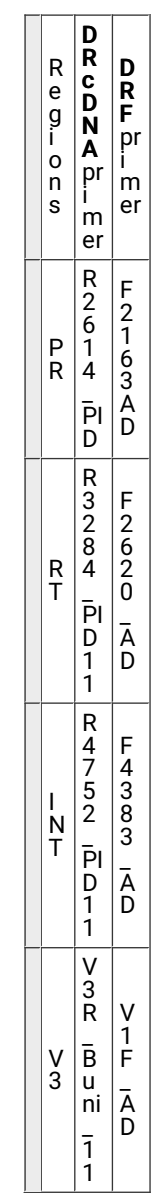

Make $10 \mu \mathrm{M}$ primer mix: mix $10 \mu \mathrm{L}$ of each primer in one set and $60 \mu \mathrm{L}$ of $\mathrm{dH}_{2} \mathrm{O}$.

\section{Primer Tables}

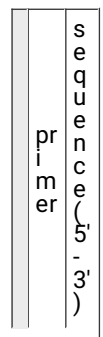




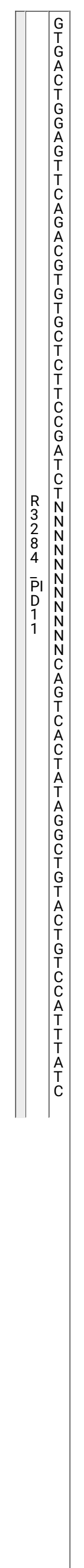

protocols.io | https://dx.doi.org/10.17504/protocols.io.sjdeci6 


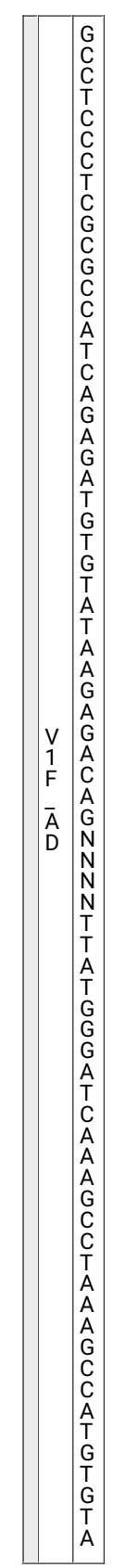

"N" in this Primer table is a random nucleotide. 


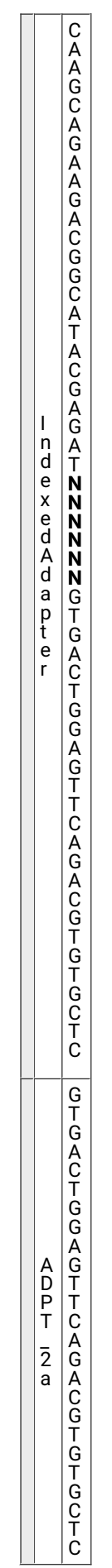

The 6 "N"s are not random nucleotides. They are a set of 24 pre-designed indexed sequences.

\section{cDNA synthesis}

3

Pipette the following components into a $0.5 \mathrm{ml}$ RNase-free tube: 


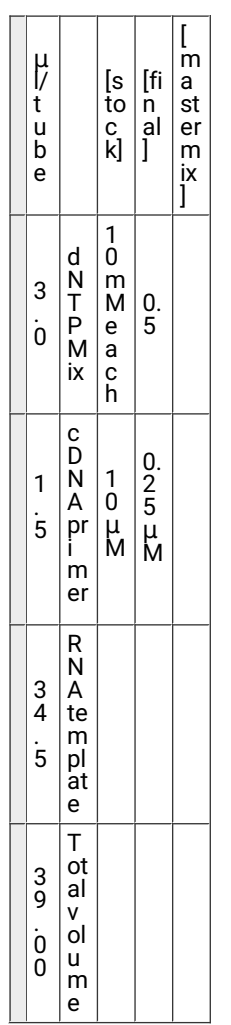

\section{\ $3 \mu \mathrm{L}$ dNTPMix \\ $\triangle 1.5 \mu \mathrm{L}$ cDNA primer \\ \ $34.5 \mu \mathrm{L}$ RNA template}

4 Place tube in $65^{\circ} \mathrm{C}$ heat block for $3-5^{\prime}$

(1) 00:05:00 $65^{\circ} \mathrm{C}$ heat block

Place the tube on ice for 1 '.

\section{(1) 00:01:00 on ice}

6

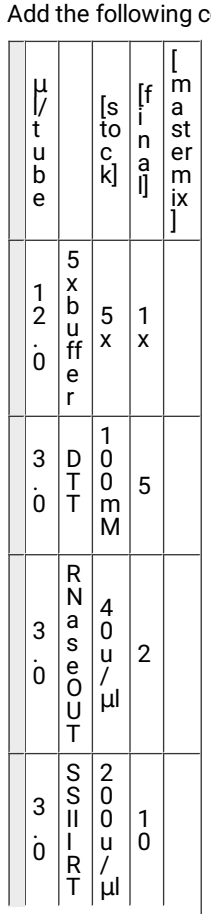




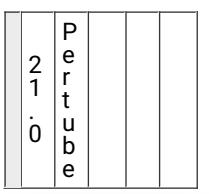

ऽ $12 \mu \mathrm{L} 5 \mathrm{x}$ buffer

$\triangle 3 \mu \mathrm{L}$ DTT

ת $3 \mu \mathrm{L}$ RNaseOUT

\ $3 \mu \mathrm{LSSIIRT}$

7 Mix and incubate at $50^{\circ} \mathrm{C}$ for $1 \mathrm{hr}$.

(1) 01:00:00 Incubation at $50^{\circ} \mathrm{C}$

8 Increase to $55^{\circ} \mathrm{C}$ and incubate for $1 \mathrm{hr}$.

(1) 01:00:00 Incubation at $55^{\circ} \mathrm{C}$

9 Inactivate SSIII RT by heating at $70^{\circ} \mathrm{C}$ for $15^{\prime}$.

(1) 00:15:00 Heating at $70^{\circ} \mathrm{C}$

10 To each tube, add $1 \mu$ l RNase $\mathrm{H}$, incubate at $37^{\circ} \mathrm{C}$ for $20^{\prime}$.

¿ $1 \mu \mathrm{L}$ RNase $\mathrm{H}$

(1) $00: 20: 00$ Incubation at $37^{\circ} \mathrm{C}$

\section{Purification}

11 Purify cDNA using Agencourt RNAClean XP.

Resuspend the beads and take an aliquot out. Keep at room temperature for at least $\mathbf{3 0}$ minutes before use. (Should be in $1 \mathrm{ml}$ aliquots) 00:30:00 Room temperature

12

Transfer the cDNA reactions into $1.7 \mathrm{~mL}$ RNase-free tubes.

13 Resuspend the beads (Vortex). Add $42 \mu$ l of beads to $60 \mu$ I cDNA (Ratio: $0.6-0.8$ ) Agencourt RNAClean XP beads to each cDNA reaction. I $42 \mu \mathrm{L}$ beads

乃 $60 \mu \mathrm{L}$ CDNA

14 Mix the Agencourt RNAClean XP and sample thoroughly by pipette mixing 15 times. No vortexing. Let the tube incubate at room temperature for 20 minutes before proceeding to the next step.

(1) 00:20:00 Room temperature

15 Place the tube onto the magnetic tube rack for 5 minutes to separate the beads from solution. (1) 00:05:00 Magnetic tube rack

16 Slowly aspirate the cleared solution from the tube and discard. This step should be performed while the tube is situated on the rack. Do not disturb the magnetic beads, which have formed a spot on the side of the tube. 
It is important to perform this step with the tube situated on the rack. Do not disturb the separated magnetic beads. Be sure to remove all of the ethanol from the bottom of the well as it may contain residual contaminants.

\section{0:00:30 Incubation at room temperature}

18 Dispense $500 \mu \mathrm{L}$ of $70 \%$ ethanol into the tube and incubate for $\mathbf{3 0}$ seconds at room temperature. Aspirate out the ethanol and discard. (2/3)

It is important to perform this step with the tube situated on the rack. Do not disturb the separated magnetic beads. Be sure to remove all of the ethanol from the bottom of the well as it may contain residual contaminants.

00:00:30 Incubation at room temperature

19 Let the reaction tube air-dry 10 minutes on the rack with the cap open. The tube(s) should air-dry until the last visible traces of ethanol evaporate. Over drying the sample may result in a lower recovery.

00:10:00 Air-drying

20 (Optional for clinical samples) Elute in $60 \mu \mathrm{l}$ water. Repeat step 12 to 19.

EO go to step \#13 undefined

21 Remove the tube from the rack and resuspend beads in $24 \mu \mathrm{l}$ DNase-free water by pipetting up and down. Place tube back on the rack and leave for 3 minutes.

¿ $24 \mu \mathrm{L}$ DNase-free water

(3) 00:03:00 Magnetic rack

\section{PCR 1}

24

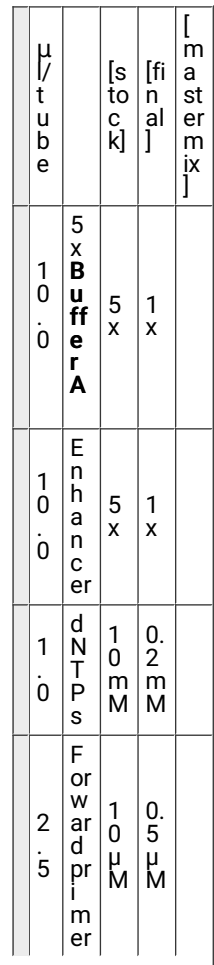




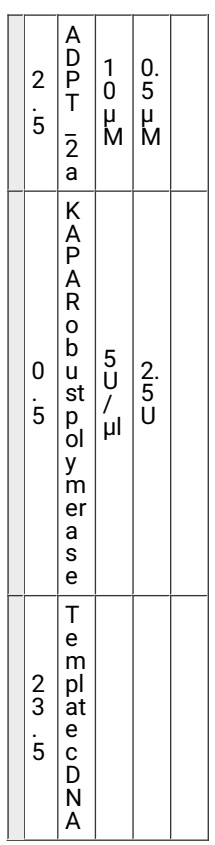

Prepare mastermix in cold box and use repeater pipette to add to each tube in the cold box. Add template cDNA to each tube and pipette up and down to mix. ¿ $10 \mu \mathrm{L}$ 5x Buffer A

\section{I $10 \mu \mathrm{L}$ Enhancer}

ת $1 \mu \mathrm{L}$ dNTPs

\ $2.5 \mu \mathrm{L}$ Forward primer

\ $2.5 \mu$ L ADPT_2a

$\triangle 0.5 \mu \mathrm{L}$ KAPA Robust polymerase

\ $23.5 \mu \mathrm{L}$ Template cDNA

25

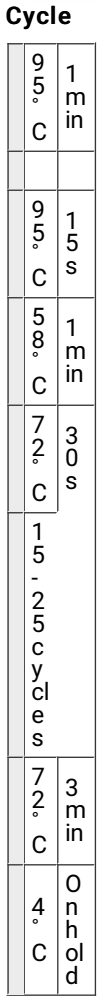

\section{Purification PCR products}

26 Purify PCR products using AmpureXP PCR cleanup kits.

Vortex the $1 \mathrm{ml}$ aliquot and remove the needed volume. Keep at room temperature for at least $\mathbf{3 0}$ minutes before use.

(1) 00:30:00 Room temperature 


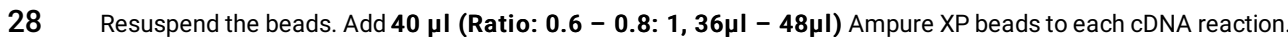
¿ $40 \mu \mathrm{L}$ Ampure XP beads

29 Mix the Ampure XP and sample thoroughly by vortexing. Let the tube incubate at room temperature for 5 minutes before proceeding to the next step (incube off the rack).
(1) 00:05:00 Incubation at room temperature

Place the tube onto the magnetic tube rack for 5 minutes to separate the beads from solution. (1) 00:05:00 Incubation magnetic rack

31 Slowly aspirate the cleared solution from the tube and discard. This step should be performed while the tube is situated on the rack. Do not disturb the magnetic beads, which have formed a spot on the side of the tube.

32 Dispense $500 \mu \mathrm{L}$ of $70 \%$ ethanol into the tube and incubate for $\mathbf{3 0}$ seconds at room temperature. Aspirate out the ethanol and discard. (1/2)

$$
\text { Note }
$$

It is important to perform this step with the tube situated on the rack. Do not disturb the separated magnetic beads. Be sure to remove all of the ethanol from the bottom of the well as it may contain residual contaminants.

\section{I $500 \mu \mathrm{L} 70 \%$ ethanol \\ 00:00:30 Incubation at room temperature}

33 Dispense $500 \mu \mathrm{L}$ of $70 \%$ ethanol into the tube and incubate for 30 seconds at room temperature. Aspirate out the ethanol and discard. (2/2)

$$
\text { Note }
$$

It is important to perform this step with the tube situated on the rack. Do not disturb the separated magnetic beads. Be sure to remove all of the ethanol from the bottom of the well as it may contain residual contaminants.

S $500 \mu \mathrm{L} 70 \%$ ethanol

$$
\text { 00:00:30 Incubation at room temperature }
$$

34 Let the reaction tube air-dry $\mathbf{1 0}$ minutes on the rack with the cap open. The tube(s) should air-dry until the last visible traces of ethanol evaporate. Over drying the sample may result in a lower recovery.

\section{(1) 00:10:00 Air-drying}

35 Remove the tube from the rack and resuspend beads in $50 \mu$ DNase-free water by pipetting up and down. Place tube back on the rack and leave for 3 minutes.

¿ $50 \mu \mathrm{L}$ DNase-free water

(1) 00:03:00 Magnetic rack

\section{PCR 2}

37

Complete thaw and vortex KAPA reagents (except for enzyme) before use. 
38

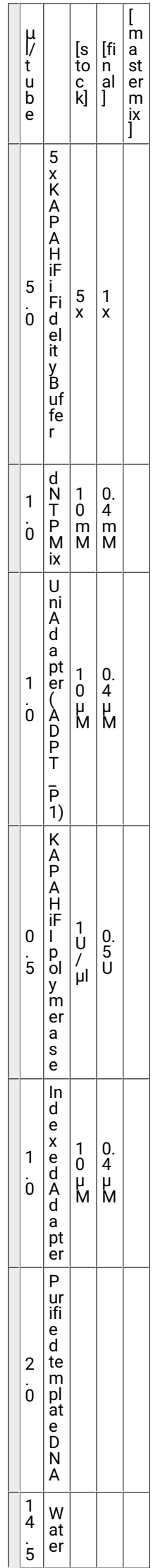

Prepare mastermix in cold box and use repeater pipette to add to each tube in the cold box. Add Indexed Adapter to each tube. 


\section{¿ $5 \mu \mathrm{L}$ 5x KAPA HiFi Fidelity Buffer \\ $\triangle 1 \mu \mathrm{L}$ dNTP Mix \\ \ $1 \mu \mathrm{L}$ Uni Adapter (ADPT_P1) \\ \ $0.5 \mu \mathrm{L}$ KAPA HiFI polymerase \\ $\triangle 1 \mu \mathrm{L}$ Indexed Adapter \\ \ $2 \mu \mathrm{L}$ Purified template DNA \\ I $14.5 \mu \mathrm{L}$ Water}

Cycle (PCR machine \#5 SZ -> ILM2):

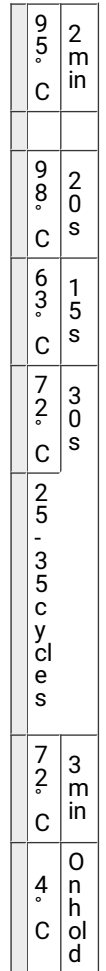

\section{Gel Purification and quantification}

40 Before gel purification, run $2 \mu$ l products on $1 \%$ agarose gel to check the bands.

41 Gel purification. (Qiagen QIAquick gel extraction kit)

Run $2^{\text {nd }}$ round $P C R$ products on $1.2 \%$ agarose gel. $E=4 \mathrm{~V} / \mathrm{cm}, T=60 \mathrm{~min}$.

Excise DNA fragment.

43

Weight the gel; add 3 volume of Buffer QG to 1 volume of gel.

44

Incubate at $50^{\circ} \mathrm{C}$ for $\mathbf{1 0}$ minutes to completing dissolve. Vortex every 2-3 minutes to help dissolve.

(1) 00:10:00 Incubation at $50^{\circ} \mathrm{C}$

(1) 00:02:30 Vortex 
46 Place MinElute column, apply the sample to the column and centrifuge for 1 minute.

00:01:00 Centrifugation

47 Add $500 \mu$ l buffer QG and centrifuge for 1 minute.

¿ $500 \mu \mathrm{L}$ Buffer QG

00:01:00 Centrifugation

48 Add $0.75 \mathrm{ml}$ buffer PE, incubate for 5 minutes at room temperature, centrifuge for 1 minute

(1) 00:05:00 Incubation at room temperature

(1) 00:01:00 Centrifugation

49 Discard the fluid, centrifuge for additional 3 minutes.

(7) 00:03:00 Centrifugation

50 Put the column in a new $1.7 \mathrm{ml}$ tube, add $\mathbf{1 0} \mu \mathrm{l}$ buffer EB. Stand for $\mathbf{4}$ minutes, centrifuge for $\mathbf{2}$ minutes.

(1) 00:04:00 Stand

51 Quantification using Invitrogen Qubit dsDNA BR Assay kit. See Qubit dsDNA BR assay protocol. Don't use Nanodrop to quantify!

Quantify the pooled library using Qubit dsDNA BR assay kit. Check the quality of the library by Agilent Bioanalyzer or Bio-rad Experion. Repeat purification if primer dimmer is present ( 200bp). 